Gut, 1968, 9, 222-226

\title{
Carcinoma of the duodenum
}

\author{
LOUIS KREEL AND COLIN MACKINTOSH
}

From the Royal Free Hospital, Gray's Inn Road, London

Primary malignant neoplasms of the duodenum are relatively uncommon, some 602 authentic cases having been reported up to 1961 (Iovine and Tsangaris, 1961). Since that date few further cases have been reported in the British literature and this paper records four recent cases.

\section{HISTORY}

A case of rupture or perforation of the duodenum was described by Hamberger in 1746; this may have represented a primary carcinoma but a benign duodenal ulcer seems the more probable pathology.

\section{INCIDENCE}

Ewing (1931) states that the incidence of malignant tumours of the small intestine is $3 \%$ of neoplasms of the gastrointestinal tract. Shallow, Eger, and Carty (1944) reported the incidence of primary carcinoma of the duodenum in a study of 350,286 necropsies as $0.03 \%$; the duodenum is affected in $45.6 \%$ of cases of small intestinal neoplasms. In this series care was taken to exclude undifferentiated neoplasms of the ampulla of Vater and of the biliary and pancreatic systems, all of which are mixed with the figures for the incidence of duodenal carcinoma throughout its history. Ficarra and Marshall (1945) state that in order of frequency the duodenum is the commonest site for carcinoma of the small bowel if carcinoma of the ampullary region is included, and hence special attention must be paid to the pathology to establish the primary site and obtain true figures for its incidence.

Spinazzola and Gillesby (1963) give the distribution of duodenal neoplasms for the first part of the supraampullary region as $18-23 \%$, for the second part as $50-65 \%$, and for the third part as $15-20 \%$. In their review of the tumour registry at the Veterans Administration Hospital, Hines, Illinois, from 1931 to 1960 inclusive, they found 31 cases of small bowel neoplasm. Of these, $39 \%$ (12 cases) were in the duodenum and only authenticated cases were included; eight of the 12 were adenocarcinomas (three carcinoid and one leiomyosarcoma). Most of the tumours were of the polypoid or ulcerating type. The incidence in males is approximately twice that in females, the average time between the onset of symptoms and diagnosis six months (Shallow, Eger, and Carty, 1945) to 11 months (Dixon, Lichtman, and Weber, 1946). These neoplasms occur most frequently in the fifth and sixth decades.

\section{CLINICAL FEATURES}

In general upper abdominal pain and weight loss are the outstanding symptoms, and usually they are not suggestive of ulcer (Spinazzola and Gillesby, 1963); in the late phases of the disease a variety of symptoms and signs have been reported, commonly those of developing high intestinal obstruction and jaundice, haematemesis, melaena, and occult blood in the stool. A variety of other findings, such as low back pain and alteration in bowel habit, have also been described.

\section{RADIOLOGICAL}

Johnson (1948) has summarized the principal radiological features of duodenal tumours as annular infiltrating or ulcerative defects, blurring and thickening of the mucosa, irregular polypoid filling defects that narrow the lumen, dilatation of the duodenum above a constricted area and of the stomach with gastric retention, and rigidity of the duodenal wall. To these should be added hyperperistaltic activity of the stomach and abnormal dilatability of the pyloric canal as usually occurs in 'pyloric stenosis' due to cicatricial narrowing of the duodenal cap.

\section{INVESTIGATIONS}

The cardinal investigation is the barium meal done in a routine manner with meticulous examination of the entire duodenum (Harper, 1967). This is of particular importance when the presenting symptoms are epigastric pain and vomiting or when gastric hyperperistalsis is present and no obstructive lesion is detected in the gastric antrum, pylorus, or duodenal cap. Failure to examine the entire duodenum adequately is one of the main reasons for late 
definitive diagnosis. Any suspicious area in the duodenum should again be examined by gas distension duodenography by which the earliest changes can be accurately assessed (Raia and Kreel, 1966).

\section{TREATMENT}

Interest in the surgical treatment of carcinoma of the ampullary region was stimulated by Whipple, Parsons, and Millins (1935), although surgery in carcinoma of the duodenum not involving the ampullary region has given discouraging results (Spinazzola and Gillesby, 1963). Berger and Koppelman (1942) reported 76 patients in whom carcinoma of the duodenum had been resected, and showed a five-year survival rate of $5 \cdot 2 \%$. Nevertheless surgery is the only hope of cure in these cases. The importance of accurate radiological examination is obvious, as is illustrated in the following case reports.

CASE 1 A man aged 60 first presented on 18 May 1965 with a history of intermittent abdominal discomfort for nine months before consultation. A barium meal in February 1965 was reported as showing no lesion. The patient had attended a dental clinic on account of diffuse dental caries when the $\mathrm{Hb}$ was found to be $42 \%$. After a full investigation iron-deficiency anaemia was diagnosed and the patient was prescribed ferrous gluconate and ascorbic acid. He remained well during one week's observation and was discharged home.

He was readmitted on 16 March 1966 complaining of anorexia and intermittent vomiting. The patient stated that he vomited every two or three weeks, the vomitus consisting of ingested food and apparently containing bile. This had been occurring for about six months accompanied by a dull epigastric ache which came on about 20 minutes after a meal and was not relieved by antacids. It was reported that he had had several barium meals during 1965 which showed no abnormality. The significant blood investigations were a $\mathrm{Hb}$ of $71 \%$ and blood urea level of $134 \mathrm{mg} \%$.

A barium meal was performed and showed a stricture of the third part of the duodenum which was reported as a carcinoma. Laparotomy was performed on 6 April 1966 and a localized stricture of the third part of the duodenum was resected, $12 \mathrm{~cm}$ of the small bowel being removed. An end-to-end anastomosis of the duodenal stump to the jejunum was made. There was no evidence of any secondary tumour. The patient made a successful recovery, and at a follow-up visit on 2 June 1966 was progressing satisfactorily.

The pathological report was of an annular stricture of the duodenum involving $3 \mathrm{~cm}$ of mucosa. This was a primary carcinoma of the duodenum which was penetrating the muscle wall and had metastasized to a regional lymph node included in the specimen.

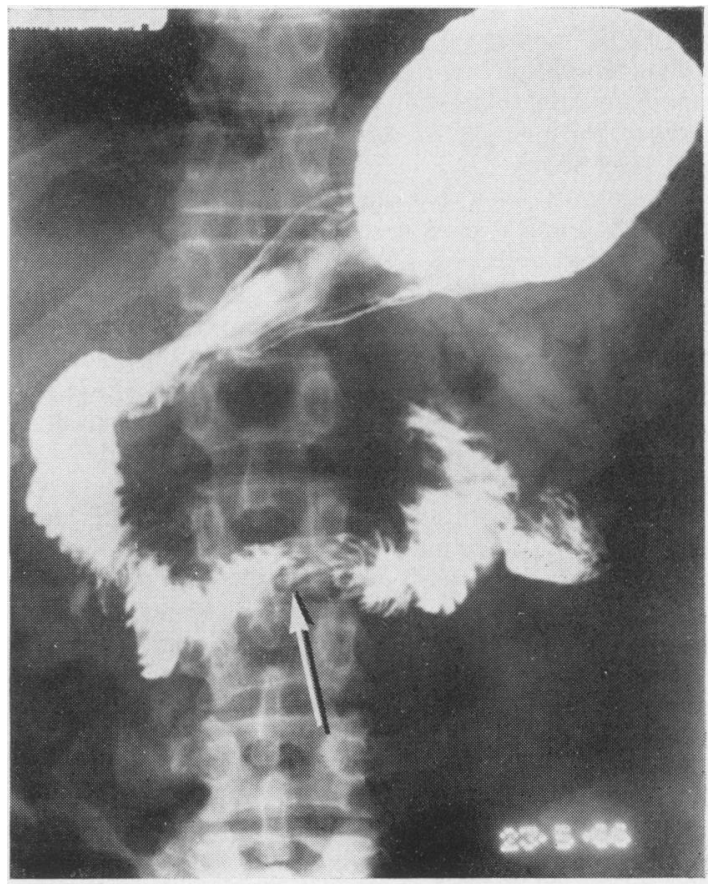

FIG. 1a.

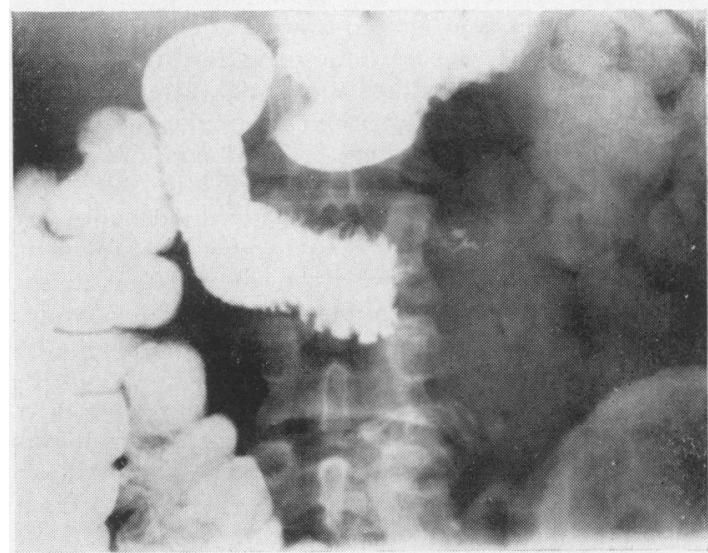

FIG. $1 b$.

FIG. 1. Case 1. (a) Mucosal distortion with slight narrowing in the third part of the duodenum as it lies on the body of the second lumbar vertebra; (b) later film showing hold up of the barium at the site of the duodenal carcinoma.

CASE 2 A woman aged 68 years presented in December 1965 with a history of aching pain in the epigastrium occurring about an hour after meals present for about six months. The pain did not radiate to the back, and was relieved by alkalis initially. Recent severe weight loss and also nausea and occasional vomiting were present. 
The patient was emaciated and debilitated. She had a history of 'cholecystitis' in 1946, and was diagnosed as having a non-functioning gall bladder in 1954. Significant blood investigations showed: Hb $65 \%$, ESR $30 \mathrm{~mm} / \mathrm{hr}$, alkaline phosphatase 16, serum amylase 450 units, blood urea level $48 \mathrm{mg} \%$.

The report of a barium meal stated: 'Oesophagus normal, stomach gross gastric dilatation with stasis and marked fluid and food residue. An obstructive lesion in the second lin. of the first part of the duodenum is present and a partly calcified opacity lies in this region which may be a gall stone.' An intravenous cholangiogram did not visualize the biliary tree but a gall stone was again noted. A preoperative diagnosis of an obstructing tumour of the duodenum was made; the possibility of a gall stone having ulcerated into the duodenum was suggested in view of the relationship of the gall stone opacity to the duodenum.

Laparotomy was performed on 29 November 1965. At operation a mass involving the duodenum, gall bladder, and splenic flexure of the colon was found, and loops of large and small bowel were adherent to the mass. A lymph node biopsy was taken and gastroenterostomy performed.

Post-operatively the patient deteriorated, she developed abdominal distension, and in view of the increasing signs of obstruction and failure to respond to conservative measures, a second laparotomy was performed on 5 December. A localized abscess was found in the right iliac fossa. The stomach was distended and free fluid was present in the peritoneal cavity; the gastroenterostomy was intact, and an ileo-transverse colostomy was performed. After the second operation the patient rapidly deteriorated and died some hours later.

At post-mortem examination a tumour mass was found encircling and invading the duodenum, ulcerating into the gall bladder, and extending into the porta hepatis; there was a gall stone in the duodenum; pus was located in the tumour mass and a purulent peritonitis was present. On naked-eye examination it appeared more likely that the primary was in the duodenum. The first biopsy report had shown a well differentiated adenocarcinoma of gastrointestinal origin, the histological appearances being reported as suggestive of a colonic rather than gastric primary. Histological examination of slides taken at necropsy again showed an adenocarcinoma, and the weight of evidence was for a primary tumour of duodenal origin.

CASE 3 A man aged 48 years initially complained of an unproductive cough which started in March 1964, shortly after he had received an anti-cholera injection; the cough subsequently became worse. A diagnosis of bronchitis was made and a course of antibiotics given. Subsequently his general health deteriorated, intermittent vomiting occurred, and the patient complained of attacks of lower chest pain. At a second consultation a nasopharyngitis was noted and a further course of antibiotics given, but the vomiting increased and the patient was referred for a surgical opinion. At this time the patient had lost 2 stone in weight. Blood tests showd $\mathrm{Hb} 13.9 \mathrm{~g} /$ $100 \mathrm{ml}$, ESR $20 \mathrm{~mm} / \mathrm{hr}$, alkaline phosphatase level

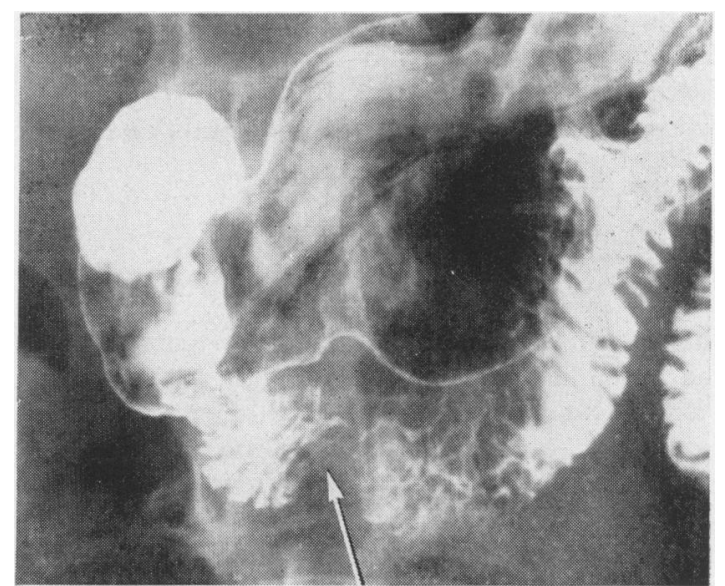

FIG. 2. Case 3. Area of narrowing of the third part of the duodenum with obliteration of the mucosal pattern.

15 K.A. units, and no other significant abnormality. A chest radiograph showed 'streaky ill-defined opacities in both middle and lower zones, most marked in the central zone. The central distribution was suggestive of azotaemic lung or lymphatic dissemination but the appearances were not typical and some form of fairly widespread lobar pneumonia seemed more likely, possibly associated with basal bronchitis.

A barium meal was reported showing a quantity of resting secretion in the stomach; the site of the obstruction appeared to be at the junction of the second and third parts of the duodenum, where there was a small stricture and possibly some ulceration. This had the appearances of a carcinoma of the duodenum.

A laparotomy was performed on 18 June 1964 and a large mass found involving the third and fourth parts of the duodenum; the pancreas was indurated, the gall bladder distended, and the stomach dilated. Multiple secondaries were present throughout the peritoneal cavity, one of which was excised from the greater omentum for biopsy, and cholecystenterostomy and gastroenterostomy were performed.

After the operation the patient's general condition deteriorated, and the wound broke down on 27 June. During repair of the abdominal wound cardiac arrest occurred and the patient could not be resuscitated.

The biopsy report on the secondary was of undifferentiated carcinoma. The pathologist stated that this would be of adenocarcinomatous origin but also a "very anaplastic squamous carcinoma must be considered'.

CASE 4 A man aged 72 years presented on 31 August 1966 with a history of intermittent abdominal cramp-like pains for four months not related to food. He complained of loss of appetite and attacks of nausea, but had only vomited about four times in the past four weeks; he had lost about 2 stones in weight in four months. There was no evidence of jaundice or other significant findings on examination. Blood examination showed $\mathrm{Hb} 9 \cdot 2 \mathrm{~g} / 100 \mathrm{ml}$, 


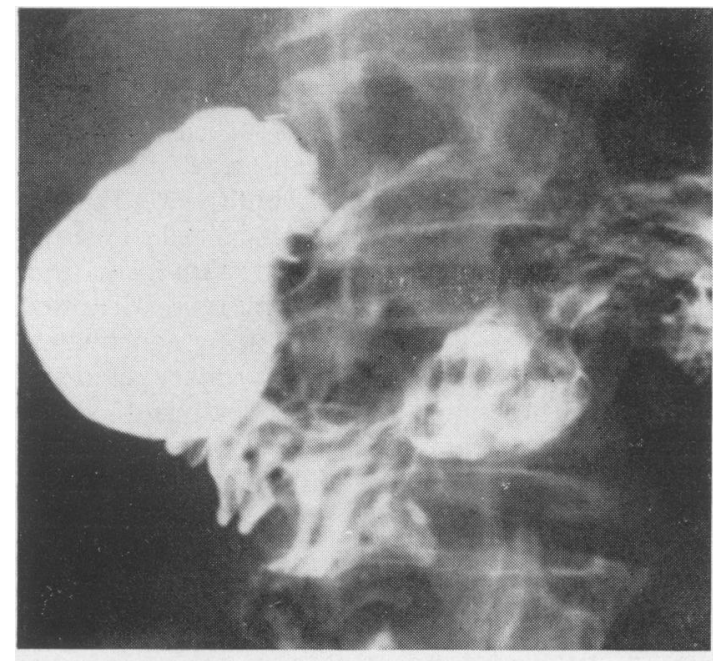

FIG. 3a.

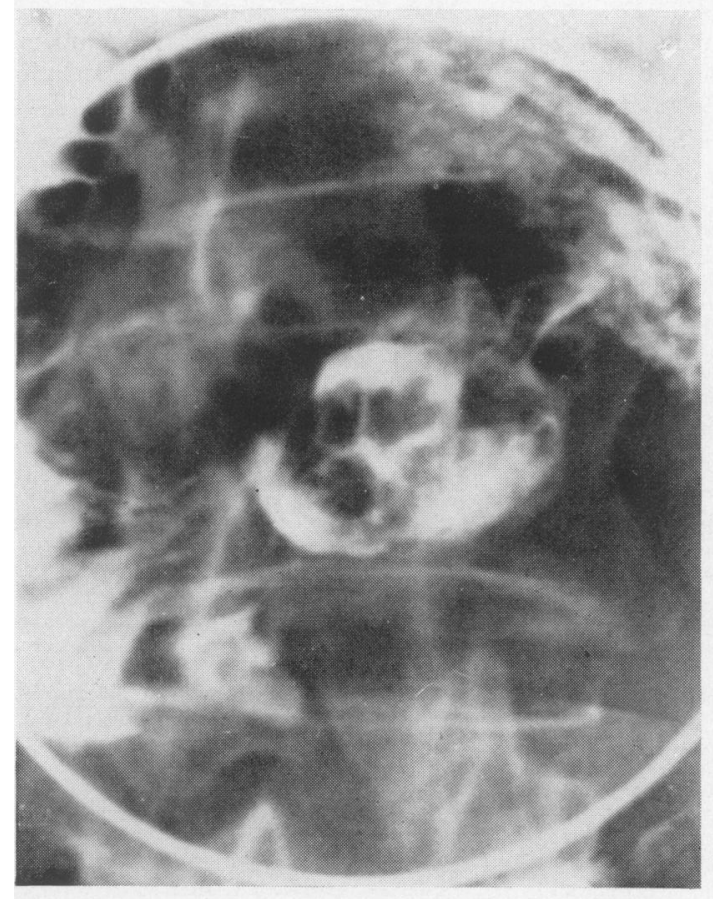

FIG. $3 b$.

FIG. 3. Case 4. (a) There are two areas of narrowing in the ascending part of the duodenum with a space-occupying lesion between: $(b)$ spot film of the tumour region showing a radiating pattern of ulceration of the tumour.

ESR $142 \mathrm{~mm} / \mathrm{hr}$, and blood urea $27 \mathrm{mg} \%$. A chest radiograph showed no active pulmonary lesion.

The result of a barium meal was reported as carcinoma of the third part of the duodenum associated with active ulceration. Laparatomy was performed on 9 September 1966 and a large mass was found in the region of the head of the pancreas and a localized lesion with narrowing of the third part of the duodenum with some proximal dilatation of the duodenum. Multiple hepatic metastases were present with carcinomatous infiltration of the omentum and duodenal lymphatics. A biopsy was taken from a liver secondary and gastroenterostomy performed. Four days postoperatively the wound broke down, and after repair the patient recovered slowly and was subsequently transferred for convalescence.

The biopsy report was 'tumour columnar celled carcinoma replacing hepatic parenchyma'.

\section{DISCUSSION}

Though carcinoma of the duodenum is a relatively rare lesion, the two main criteria of success in early diagnosis are awareness of the possibility of the lesion and meticulous examination of the duodenum at all barium meal examinations. Suspicious areas in the duodenum should be re-examined by gas distension duodenography with special attention to early mucosal changes, as radical surgery is the only hope of cure. The radiologist plays a central role in early diagnosis as no other effective diagnostic procedure can be brought to bear on the duodenum in early cases with indeterminate symptoms. Although taking a careful history shows that in general the symptoms are not similar to those of peptic ulcer, these patients tend to be treated as cases of the ulcer diathesis until the later stages of the disease. It is simple to diagnose pyloric stenosis radiologically, but it is essential to establish accurately the nature and level of the duodenal obstruction as this gives the surgeon valuable preoperative information. If an obstructive lesion is in the first part of the duodenum at the level of the cap, there will be in the great majority of cases cicatrical narrowing due to peptic ulceration; when the lesion is beyond the cap, in the absence of stenosing postbulbar ulcer, other lesions must be considered, such as duodenal web, intraduodenal diverticulum, extrinsic lesions, and carcinoma of the duodenum.

As the incidence rates show, the pathological evaluation of the origin of obstructing duodenal lesions is fraught with argument, hence the marked difference in incidence in various series of small bowel neoplasms of primary duodenal tumours.

The second, third, and fourth cases in our series illustrate the point that careful attention must be paid to the investigative findings before final interpretation and acceptance of the histological report. In the third case, a man of 48 with a probable pulmonary lymphangitis carcinomatosa, a clear duodenal mucosal lesion was demonstrated. The biopsy report was of undifferentiated carcinoma in 
a secondary nodule, and it must be accepted that the weight of evidence is for a duodenal primary with secondary spread, as virtually no other sequence of events could explain the radiological picture of the duodenum. Similarly, in the case of the woman with a gall stone in the duodenum and a localized associated stricture visible on naked-eye examination arising from the duodenum and encircling it. There was also ulceration into the gall bladder and extension into the porta hepatis. The biopsy showed an adenocarcinoma and again the evidence pointed to a duodenal primary.

The fourth case is an excellent example of a duodenal ulcerative lesion demonstrated at barium meal examination, and at laparotomy a large mass was found in the region of the head of the pancreas with a localized lesion of the duodenum. A biopsy showed columnar celled carcinoma from a secondary. It is most unlikely that a primary neoplasm of the pancreas would produce a localized lesion of the third part of the duodenum with mucosal changes as shown on the barium meal rather than a widespread involvement of the duodenum. Furthermore, localized mucosal lesions, such as those demonstrated in this case in the third part of the duodenum, have not been described in carcinoma of the head of the pancreas, the usual changes being found in the region of the ampulla and commonly associated with obstructive jaundice.

The assessment of the primary site in three of the cases rested largely on the radiological findings of localized mucosal change in the duodenum with varying degrees of stricture, in association with a biopsy of a secondary deposit showing adenocarcinoma. These findings can only be adequately correlated with the clinical and pathological evidence in three cases by a diagnosis of primary duodenal carcinoma.

\section{SUMMARY}

The history and incidence of carcinoma of the duodenum have been summarized and the radiological features and results of treatment reviewed. Four cases of carcinoma of the duodenum are reported, one illustrating delay in diagnosis due to initial inadequate duodenal examination. Another presented with radiological features of 'pyloric stenosis' in the initial stage of the barium examina- tion, but in pursuing the examination, an obstructive lesion of the first part of the duodenum distal to the cap was demonstrated with a closely related gall stone. A preoperative diagnosis of carcinoma of the duodenum with possible gall stone obstruction was made and subsequently confirmed. The remaining two cases showed localized mucosal changes with varying degrees of stricture formation, and a biopsy report of secondary lesions showed adenocarcinoma, the findings being adequately correlated only by a diagnosis of primary duodenal carcinoma. The importance of meticulous duodenal examination at barium meal studies was emphasized and the value of gas-distension double-contrast duodenography in early diagnosis is indicated.

We wish to thank the surgeons and physicians who referred these cases for radiological investigation, particularly Mr. George Qvist for his cooperation and encouragement. We should also like to thank Professor Sherlock and the Medical Unit of the Royal Free Hospital, Dr. Symons, Miss George, and Mr. Gracey for access to the clinical notes, and Mr. Gilson and members of the Photographic Department, The Royal Free Hospital, for the illustrations.

\section{REFERENCES}

Berger, L., and Koppelman, H. (1942). Primary carcinoma of the duodenum. Ann. Surg., 116, 738-750.

Dixon, C. F., Lichtman, A. L., and Weber, H. M. (1946). Malignant lesions of the duodenum. Surg. Gynec. Obstet., 83, 83-93.

Ewing, J. (1931). Neoplastic Disease, 3rd ed., p. 704. Saunders, Philadelphia.

Ficarra, B. J., and Marshall, S. F. (1945). Primary carcinoma of the jejunum. Surg. Clin. N. Amer., 25, 713-718.

Hamberger, G. E. (1746). De Ruptura Intestini Duodeni. Also Ritter, Jena (1757). In Haller, A. Von. Disputationes ad Morborum Historiam et Curationem Facientes, vol. 3, pp. 507-509. M. M. Bousquet, Lausanne.

Harper, R. A. K. (1967). Radiology of the Duodenum, p. 122. Lloyd Luke, London.

Iovine, V. M., and Tsangaris, N. (1961). Primary carcinoma of the duodenum. Amer. Surg., 27, 744-750.

Johnson, J. B. (1948). Report of case of primary papillary adenocarcinoma of duodenum with special reference to X-ray study. Tex. Rep. Biol. Med., 6, 373-378.

Raia, S., and Kreel, L. (1966). Gas-distension, double-contrast duodenography using the Scott-Harden gastroduodenal tube. Gut, 7, 420-424.

Shallow, T. A., Eger, S. A., and Carty, J. B. (1944). Primary carcinoma of third portion of duodenum. Surgery, 16, 939-946.

(1945). Primary malignant disease of the small intestine. Amer. J. Surg., 69, 372-383.

Spinazzola, A. J., and Gillesby, W. J. (1963). Primary malignant neoplasms of the duodenum: report of 12 cases. Amer. Surg., 6, 405-412.

Whipple, O. A., Parsons, W. B., and Millins, C. R. (1935). Treatment of carcinoma of the ampulla of Vater. Ann. Surg., 102, 763779. 